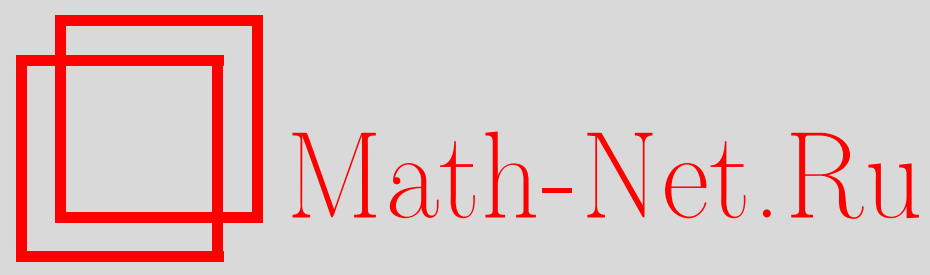

В. А. Ватутин, Асимптотические свойства числа инверсий в случайном лесе, Матем. вопр. криптогр., 2020, том 11, выпуск 4, 7-22

DOI: https://doi.org/10.4213/mvk337

Использование Общероссийского математического портала Math-Net.Ru подразумевает, что вы прочитали и согласны с пользовательским соглашением http://www . mathnet.ru/rus/agreement

Параметры загрузки:

IP : 54.81 .137 .203

26 апреля 2023 г., 02:41:47

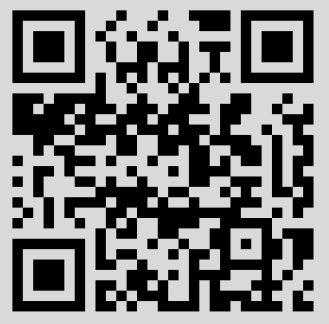


МАТЕМАТИЧЕСКИЕ ВОПРОСЫ КРИПТОГРАФИИ

2020 T. 11 № 4 C. 7-22

УДК 519.212.2+519.218.23

DOI https://doi.org/10.4213/mvk337

\title{
Асимптотические свойства числа инверсий в случайном лесе
}

\author{
В. А. Ватутин \\ Математический институт им В.A. Стеклова \\ Российской академии наук, Москва
}

Получено 15.V.2020

Аннотация. Пусть $\mathcal{F}_{n, N}=\left\{F_{n, N}\right\}-$ множество всех лесов, каждый из которых образован $N$ помеченными корневыми деревьями $T_{1}, T_{2}, \ldots, T_{N}$, имеющими в совокупности $n+N$ вершин (считая корни), причем вершинам приписаны несовпадающие метки (числа от 1 до $n+N)$. Обозначим $\lambda(u)$ метку, приписанную вершине $u$ леса $F_{n, N}$. Будем говорить, что вершина $u$ дерева $T_{i}$ является предком вершины $v$ того же дерева и писать $u \prec v$, если путь, ведущий по ребрам от корня дерева к вершине $v$, проходит через вершину $u$. Числом инверсий $\mathcal{I}\left(F_{n, N}\right)$ в лесе $F_{n, N}$ называется число таких пар вершин $u, v$ в этом лесе, что $u \prec v$ и $\lambda(u)>\lambda(v)$. При условии, что лес $F_{n, N}$ выбирается случайно и равновероятно из множества $\mathcal{F}_{n, N}$, найдено предельное распределение случайной величины $n^{-3 / 2} \mathcal{I}\left(F_{n, N}\right)$ при $n \rightarrow \infty$ и $2 N n^{-1 / 2} \rightarrow l \in[0, \infty)$.

Ключевые слова: случайный лес, высота случайного леса, инверсия, предельная теорема

\section{Asymptotic properties of the number of inversions in a random forest}

\section{A. Vatutin}

Steklov Mathematical Institute of Russian Academy of Sciences, Moscow

Abstract. Let $\mathcal{F}_{n, N}=\left\{F_{n, N}\right\}$ be the set of all forests each of which is generated by $N$ labelled rooted trees $T_{1}, T_{2}, \ldots, T_{N}$ having in total $n+N$ vertices (including the roots) with different labels (numbers from 1 to $n+N)$. Denote by $\lambda(u)$ the label assigned to the vertex $u$ of the forest $F_{n, N}$. We say that a vertex $u \in T_{i}$ is an ancestor of a vertex $v \in T_{i}$ and write $u \prec v$, if the path leading from the root of the tree to the vertex $v$ passes the vertex $u$. The number of inversions of a forest $F_{n, N}$ is the number of pairs of vertices $u, v$ in this forest such that $u \prec v$ and $\lambda(u)>\lambda(v)$. Assuming that $F_{n, N}$ is a forest selected randomly and equiprobably from

Citation: Matematicheskie Voprosy Kriptografii, 2020, v. 11, № 4, pp. 7-22 (Russian)

(C) Академия криптографии Российской Федерации, 2020 г. 
$\mathcal{F}_{n, N}$ we find the limiting distribution of the random variable $n^{-3 / 2} \downarrow\left(F_{n, N}\right)$ as $n \rightarrow \infty$ and $2 N n^{-1 / 2} \rightarrow l \in[0, \infty)$.

Keywords: random forest, the height of a random forest, inversion, limit theorem

\section{1. Введение}

В настоящей работе изучаются некоторые характеристики лесов $F_{n, N}$, состоящих из $N$ корневых деревьев, имеющих в совокупности $n+N$ некорневых и корневых вершин, занумерованных несовпадающими метками (числами от 1 до $n+N)$. В дальнейшем корневые вершины будем для краткости называть корнями. Предполагаем, что ребра деревьев направлены от корней, и считаем, что два дерева изоморфны (неразличимы), если метки их корней совпадают и между множествами их вершин существует взаимно-однозначное соответствие, сохраняющее смежность и нумерацию вершин. Отметим, что множество $F_{n, 1}$ является множеством помеченных корневых деревьев с $n$ некорневыми вершинами.

Скажем, что леса $F_{n, N}$ и $F_{n, N}^{\prime}$ изоморфны (неразличимы), если множества меток корней, входящих в них деревьев совпадают, причем деревья с одинаковыми метками корней изоморфны. Будем считать, что деревья $T_{1}, T_{2}, \ldots, T_{N}$, входящие в лес $F_{n, N}$, упорядочены согласно следующему правилу: $T_{1}$ - это дерево, содержащее вершину с меткой 1 , a $T_{k}, k \geqslant 2,-$ это дерево, содержащее вершину с минимальной меткой, не использованной при нумерации вершин деревьев $T_{1}, \ldots, T_{k-1}$.

Обозначим $\mathcal{F}_{n, N}=\left\{F_{n, N}\right\}$ множество всех неизоморфных помеченных лесов $F_{n, N}$.

Леса, принадлежащие множеству $\mathcal{F}_{n, N}$, естественным образом возникают при исследовании свойств случайных отображений (см. $[1,4,7,12])$. Известно (см., например, [12, раздел 6.2.4]), что мощность $\left|\mathcal{F}_{n, N}\right|$ множества $\mathcal{F}_{n, N}$ вычисляется по формуле

$$
\left|\mathcal{F}_{n, N}\right|=N C_{n+N}^{N}(n+N)^{n-1} .
$$

Напомним некоторые факты и определения, характеризующие такие леса и входящие в них деревья. Пусть $T$ - корневое дерево с ребрами, направленными от корня. В дальнейшем факт принадлежности вершины $u$ дереву $T$ будем записывать, допуская некоторую вольность 
в обозначениях, в виде $u \in T$. Кратностью вершины дерева будем называть количество ребер, выходящих из нее и направленных от корня. Высотой $h(u)$ вершины $u \in T$ называется количество ребер, принадлежащих пути, ведущему от корня к вершине $u$. Корень (по определению) имеет нулевую высоту. Высотой дерева $T$ называется величина

$$
h(T):=\max _{u \in T} h(u) .
$$

Через $|T|$ обозначим число вершин в дереве $T$ (включая корневую).

Будем говорить, что вершина $u \in T$ является предком вершины $v \in T$, а вершина $v$ является потомком вершины $u$, и писать $u \prec v$, если путь, ведущий по ребрам от корня дерева к вершине $v$, проходит через вершину $u$. Соотношение $u \preceq v$ означает, что либо $u \prec v$, либо $u=v$.

Если вершина $u \in T$, то через $T(u)$ будем обозначать корневое дерево $T(u) \subset T$ с корнем $u$, вершинами, являющимися всеми потомками вершины $u$, и ребрами, соединяющими эти вершины.

Пусть $T_{1}, T_{2}, \ldots, T_{N}$ - помеченные деревья, образующие лес $F_{n, N}$. Высота леса $F_{n, N}$ задается соотношением

$$
h\left(F_{n, N}\right):=\max _{1 \leqslant i \leqslant N} h\left(T_{i}\right) .
$$

Обозначим $\lambda(u)$ метку, приписанную вершине $u \in F_{n, N}$. Рассмотрим какое-либо дерево $T_{i}, i=1,2, \ldots, N$. Скажем, что пара вершин $u \prec v$, принадлежащих дереву $T_{i}$, образует инверсию, если $\lambda(u)>\lambda(v)$.

Числом инверсий вершины $u \in T_{i}$ назовем величину

$$
M(u):=\sum_{v \in T_{i}: u \prec v} \chi\{\lambda(u)>\lambda(v)\},
$$

где $\chi\{B\}-$ индикатор события $B$. Число инверсий $\mathcal{I}\left(T_{i}\right)$ в дереве $T_{i}$ определяется соотношением

$$
\mathcal{I}\left(T_{i}\right):=\sum_{u \in T_{i}, v \in T_{i}: u \prec v} \chi\{\lambda(u)>\lambda(v)\}=\sum_{u \in T_{i}} M(u) .
$$

Числом инверсий в лесе $F_{n, N}$ назовем величину

$$
\begin{aligned}
\mathcal{I}\left(F_{n, N}\right):=\sum_{i=1}^{N} \mathcal{I}\left(T_{i}\right) & \\
& =\sum_{T_{i} \in F_{n, N}} \sum_{u \in T_{i}, v \in T_{i}: u \prec v} \chi\{\lambda(u)>\lambda(v)\}=\sum_{i=1}^{N} \sum_{u \in T_{i}} M(u) .
\end{aligned}
$$


Цель данной работы - исследовать распределение случайной величины $\mathcal{I}\left(F_{n, N}\right)$, вычисленной для леса $F_{n, N}$, выбранного случайно и равновероятно из множества $\mathcal{F}_{n, N}$. Параметры распределения случайной величины $\mathcal{I}\left(F_{n, N}\right)$ могут использоваться при анализе свойств алгоритмов поиска данных и процедур линейного хеширования (см., например, [9]).

Пусть $m_{v}=|T(v)|$ обозначает число вершин поддерева с корнем $v$. Доказательство основного результата настоящей работы существенно использует следующую лемму.

Лемма 1 (см. [8]). Если T- биксированное корневое дерево, вершины которого помечены случайным равновероятным образом числами $1,2, \ldots,|T|$ без повторений, то

$$
\mathcal{I}(T) \stackrel{d}{=} \sum_{v \in T} M(v)
$$

где случайные величины $\{M(v), v \in T\}$ независимы в совокупности, причем для каждого $v \in T$ случайная величина $M(v)$ имеет равновероятное распределение на множсестве $0,1, \ldots, m_{v}-1$.

Повторяя практически дословно доказательство этой леммы, приведенное в [8], можно убедиться в справедливости следующего утверждения.

Лемма 2. Если $F_{n, N}-\oint$ фисированный лес, образованный $N$ корневыми деревъями $T_{1}, \ldots, T_{N}$, имеюшими в совокупности $n+N$ вершин, помеченных случайным равновероятным образом числами от 1 до $n+N$ (без повторений), то

$$
\mathcal{I}\left(F_{n, N}\right) \stackrel{d}{=} \sum_{i=1}^{N} \mathcal{I}\left(T_{i}\right),
$$

где случайные величины $\left\{\mathcal{I}\left(T_{i}\right), i=1,2, \ldots, N\right\}$ независимы в совокупности и имеют представления

$$
\mathcal{I}\left(T_{i}\right) \stackrel{d}{=} \sum_{u \in T_{i}} M(u)
$$

в которых случайные величины $\left\{M(u), u \in T_{i}\right\}$ независимы в совокупности, причем для каждого $u \in T_{i}$ случайная величина $M(u)$ имеет равновероятное распределение на множестве $0,1, \ldots, m_{u}-1$. 
Пусть

$$
\Upsilon(T)=\sum_{v \in T} h(v)
$$

- совокупная длина всех путей от корня к вершинам дерева $T$, а $\Upsilon\left(F_{n, N}\right)$ - совокупная длина всех путей от корней к вершинам леса $F_{n, N}$. Нетрудно проверить, что

$$
\Upsilon\left(F_{n, N}\right)=\sum_{i=1}^{N} \sum_{v \in T_{i}} h(v)=\sum_{i=1}^{N} \Upsilon\left(T_{i}\right)=\sum_{k=0}^{n} k H_{n, N}(k),
$$

где $H_{n, N}(k)$ - число вершин высоты $k$ в лесе $F_{n, N}$.

Легко понять, что $\mathbf{P}(\lambda(u)>\lambda(v))=1 / 2$ для любых вершин $u \prec v$, принадлежащих одному и тому же дереву. Поэтому

$$
\begin{gathered}
\mathbf{E}\left[\mathcal{I}\left(F_{n, N}\right) \mid T_{1}, \ldots, T_{N}\right]=\sum_{i=1}^{N} \mathbf{E}\left[\mathcal{I}\left(T_{i}\right) \mid T_{i}\right]=\sum_{i=1}^{N} \sum_{u \in T_{i}} \mathbf{E}[M(u)] \\
=\sum_{i=1}^{N} \sum_{u \in T_{i}} \sum_{v \in T_{i}: u \prec v} \mathbf{E}[\chi\{\lambda(u)>\lambda(v)\}]=\frac{1}{2} \sum_{i=1}^{N} \sum_{v \in T_{i}} \sum_{u \in T_{i}: u \prec v} 1 \\
=\frac{1}{2} \sum_{i=1}^{N} \sum_{v \in T_{i}} h(v)=\frac{1}{2} \sum_{i=1}^{N} \Upsilon\left(T_{i}\right)=\frac{1}{2} \Upsilon\left(F_{n, N}\right) .
\end{gathered}
$$

Эта формула будет использована в доказательстве основной теоремы.

Обозначим через $\mathcal{B}:=\left\{B_{r}, r \geqslant 0\right\}$ стандартное броуновское движение с нулевым сносом, тогда $\mathbf{E}\left[B_{r} B_{t}\right]=\min (r, t)$. Пусть, далее, $\mathcal{B}^{\mathrm{br}, t}:=\left\{B_{s}^{\mathrm{br}, t}, 0 \leqslant s \leqslant t\right\}-$ броуновский мост длины $t$, построенный по $\mathcal{B}$, а

$$
\mathcal{B}^{|\mathrm{br}|, t}:=\left\{\left|B_{s}^{\mathrm{br}, t}\right|, 0 \leqslant s \leqslant t\right\}
$$

- отраженный броуновский мост длины $t$. Пусть

$$
L_{t, r}^{|\mathrm{br}|}=\lim _{\varepsilon \downarrow 0} \frac{1}{\varepsilon} \int_{0}^{t} \chi\left\{r \leqslant\left|B_{s}^{\mathrm{br}, t}\right| \leqslant r+\varepsilon\right\} d s
$$

- локальное время на уровне $r \geqslant 0$ отраженного броуновского моста длины $t$, и, наконец,

$$
\begin{aligned}
\mathcal{B}^{|\mathrm{br}|, t}(l) & :=\left\{\left|B_{s}^{\mathrm{br}, t}(l)\right|, 0 \leqslant s \leqslant t\right\} \\
& =\left\{\left|B_{s}^{\mathrm{br}, t}\right|, 0 \leqslant s \leqslant t \mid 2 L_{t, 0}^{|\mathrm{br}|}=l\right\}
\end{aligned}
$$


- отраженный броуновский мост длины $t$, локальное время в нуле которого равно $l / 2$. Описание свойств отраженного броуновского моста имеется, например, в [10] и [11].

Сформулируем основной результат данной работы.

Теорема. Если параметры п и $N=N(n)$ меняются так, что

$$
\lim _{n \rightarrow \infty} \frac{2 N}{\sqrt{n}}=l \in[0, \infty)
$$

mo при $n \rightarrow \infty$

$$
\frac{2}{n^{3 / 2}} \mathcal{I}\left(F_{n, N}\right) \stackrel{d}{\rightarrow} \int_{0}^{1}\left|B_{s}^{\mathrm{br}, 1}(l)\right| d s=: \xi(l) .
$$

Замечание 1. Распределение случайной величины $\xi(l)$ является обобщенным распределением Эйри (см. [10]). Из работы Ю.Л. Павлова [5, теорема 2] следует, что если выполнено условие (4), то для любых $x>0, l>0$

$$
\mathbf{P}(\xi(l)>x)=\frac{e^{l^{2} / 8}}{l} \sqrt{\frac{2}{\pi}} \int_{-\infty}^{+\infty} e^{-i y}\left(e^{-l \sqrt{-i y / 2}}-e^{-l d(x ; y) / 2}\right) d y,
$$

где

$$
d(x ; y):=\frac{\sqrt{-2 i y}\left(1+e^{-x \sqrt{-2 i y}}\right)}{1-e^{-x \sqrt{-2 i y}}} .
$$

Формулы для вычисления моментов любого порядка случайной величины $\xi(l)$ имеются в [11]. В частности,

$$
\mathbf{E} \xi(l)=\frac{1}{\sqrt{2}} e^{l^{2} / 8} \int_{l / \sqrt{8}}^{\infty} e^{-z^{2}} d z,
$$

что при $l=0$ дает

$$
\mathbf{E} \xi(0)=\frac{1}{2} \sqrt{\frac{\pi}{2}}
$$

Замечание 2. Данную заметку можно рассматривать как дополнение к статье [8], в которой при $n \rightarrow \infty$ было найдено предельное распределение числа инверсий в случайном дереве, имеющем равновероятное распределение на множестве всех помеченных корневых деревьев с $n$ вершинами. 
Отметим, что задача, близкая по смыслу (но не по результатам) к задаче, анализируемой в настоящей работе, рассматривалась [2]. В указанной статье каждая вершина $u$ корневого $b$-арного дерева $T$ раскрашивалась независимо от остальных вершин случайно и равновероятно в один из $m$ цветов, занумерованных буквами алфавита $\mathcal{A}=\left\{A_{1}<A_{2}<\ldots<A_{m}\right\}$. При этом считалось, что пара вершин $u \prec v$ дерева $T$ образует инверсию, если цвет $A(u)$ вершины $u$ был больше, чем цвет $A(v)$ вершины $v$, и исследовалось предельное распределение числа инверсий $b$-арного дерева $T$ при такой раскраске, когда параметры $m$ и $b$ изменяются согласованным образом.

\section{2. Вспомогательные результаты}

При доказательстве теоремы нам будет нужно оценить сверху математическое ожидание случайной величины $h\left(F_{n, N}\right)$. Для вывода желаемой оценки понадобится вспомогательный ветвящийся процесс Гальтона - Ватсона $\{Z(n), n \geqslant 0\}$ с производящей функцией $f(s)=\mathbf{E} s^{\eta}$ чисел потомков частиц.

Обозначим $T$ (случайное) дерево, получившееся в результате эволюции такого процесса. Будем интерпретировать $T$ как плоское корневое дерево, т.е. корневое дерево, вложенное в плоскость, в котором между вершинами, лежащими на одном и том же расстоянии от корня, существует линейная упорядоченность. В таком дереве частицам соответствуют вершины, причем первоначальной частице соответствует корень, а ребра дерева направлены от корня и соединяют частицыпредки с частицами-потомками.

Для задания линейной упорядоченности вершин дерева $T$ необходимо сначала присоединить к корню такого дерева фиктивную вершину ребром, направленным к корню, а затем воспользоваться следующей стандартной для теории ветвящихся процессов процедурой присваивания меток частицам-вершинам (см., например, [3], §2). Первоначальной частице присваивается метка (0), а остальным частицамвершинам - метки вида $\left(0, l_{1}, \ldots, l_{k}\right), l_{i} \in\{1,2, \ldots\}$, где вершиначастица $\left(0, l_{1}, \ldots, l_{k-1}\right)$ является предком частицы $\left(0, l_{1}, \ldots, l_{k-1}, l_{k}\right)$, а частицы $\left(0, l_{1}, \ldots, l_{k}, i\right), i=1,2, \ldots$, - ее потомками. Будем считать, что если вершины $\left(0, l_{1}, \ldots, l_{k-1}, i\right)$ и $\left(0, l_{1}, \ldots, l_{k-1}, i+1\right)$ принадлежат такому дереву, то первая из них располагается на плоскости левее второй. При этом предполагается, что фиктивная вершина расположена левее всех остальных вершин дерева $T$. Обозначим $\mathcal{T}_{q}=\{T\}$ множество всех различных реализаций ветвящегося процесса, каждая из которых 
имеет $q$ вершин.

В [6] (теорема 1.2) показано, что если $Z(0)=1, \mathbf{E} \eta=1$ и $\mathbf{D} \eta \in(0, \infty)$, а генеалогические деревья соответствующего процесса Гальтона - Ватсона интерпретируются как плоские корневые деревья, то существуют такие константы $C_{1}$ и $C_{2}$, что для всех $x>0$ и $q=1,2, \ldots$

$$
\mathbf{P}\left(h(T)>x \mid T \in \mathcal{T}_{q}\right) \leqslant C_{1} e^{-C_{2} x^{2} / q} .
$$

Нам понадобится аналогичная оценка для высоты леса, выбранного случайно и равновероятно из множества $\mathcal{F}_{n, N}$.

Рассмотрим сначала множество $\mathcal{F}_{q, 1}$ всех неизоморфных помеченных корневых деревьев с $q$ некорневыми вершинами. Из (1) следует, что мощность этого множества равна $(q+1)^{q}$. Пусть $F_{q, 1}$ - дерево, выбранное случайно и равновероятно из множества $\mathcal{F}_{q, 1}$. Обозначим $\mu_{r}\left(t, F_{q, 1}\right), r, t=0,1, \ldots, q$, число вершин высоты $t$ в $F_{1, q}$, имеющих кратность $r$, и пусть $\mu_{r}(t, T)$ - соответствующие характеристики дерева $T \in \mathcal{T}_{q}$, полученного в качестве реализации ветвящегося процесса Гальтона - Ватсона с производящей функцией числа потомков частиц

$$
f(s)=\sum_{k=0}^{\infty} \mathbf{P}(\eta=k) s^{k}=e^{s-1} .
$$

Введем матрицы $\left\|\mu_{r}\left(t, F_{q, 1}\right)\right\|$ и $\left\|\mu_{r}(t, T)\right\|$ размера $(q+1) \times(q+1)$, и пусть $\mathbf{M}=\left\|m_{r}(t)\right\|$ - матрица такого же размера, элементами которой являются неотрицательные целые числа.

Следующее утверждение является естественной модификацией леммы 1 главы II монографии [4].

Лемма 3. При всех $q=1,2, \ldots$ справедливо равенство

$$
\mathbf{P}\left(\left\|\mu_{r}\left(t, F_{q, 1}\right)\right\|=\mathbf{M}\right)=\mathbf{P}\left(\left\|\mu_{r}(t, T)\right\|=\mathbf{M} \mid T \in \mathcal{T}_{q}\right) .
$$

Из этой леммы и неравенства (5) вытекает, что существуют такие константы $C_{1}$ и $C_{2}$, что для всех $x>0$ и $q=1,2, \ldots$ справедливо неравенство

$$
\mathbf{P}\left(h\left(F_{q, 1}\right)>x\right) \leqslant C_{1} e^{-C_{2} x^{2} / q} .
$$

Приведенные соотношения позволяют доказать следующее важное утверждение для лесов, состоящих из помеченных корневых деревьев.

Лемма 4. Существует такая константа $C \in(0, \infty)$, что при всех $n$ и $N$ для математического ожидания высоты $h\left(F_{n, N}\right)$ леса $F_{n, N}$, выбранного случайно и равновероятно из множества $\mathcal{F}_{n, N}$, справедливо неравенство

$$
\mathbf{E} h\left(F_{n, N}\right) \leqslant C \sqrt{N(n+N)} .
$$


Доказательство. Из (6) следует, что найдется такая константа $C$, что

$$
\mathbf{E} h\left(F_{q, 1}\right)=\int_{0}^{\infty} \mathbf{P}\left(h\left(F_{q, 1}\right)>x\right) d x \leqslant C_{1} \int_{0}^{q} e^{-C_{2} x^{2} / q} d x \leqslant C \sqrt{q}
$$

при всех $q=1,2, \ldots$

Очевидно, что если лес $F_{n, N}=\left(T_{1}, T_{2}, \ldots, T_{N}\right)$ имеет равновероятное распределение на множестве $\mathcal{F}_{n, N}$, то на любом подмножестве $\mathcal{A} \subset \mathcal{F}_{n, N}$ условное распределение леса $F_{n, N}\left(\right.$ при условии $F_{n, N} \in \mathcal{A}$ ) также равновероятно. Пусть $F_{n, N}=\left(T_{1}, T_{2}, \ldots, T_{N}\right)$ - лес, выбранный случайно и равновероятно из подмножества всех таких лесов, принадлежащих $\mathcal{F}_{n, N}$, которые образованы деревьями $t_{1}, t_{2}, \ldots, t_{N}$, имеющими $q_{1}, q_{2}, \ldots, q_{N}$ вершин соответственно. Легко понять, что при таком случайном выборе леса для каждого $i=1,2, \ldots, N$ распределение высоты дерева $T_{i}$ совпадает с распределением высоты дерева, выбранного случайно и равновероятно из множества $\mathcal{F}_{q_{i}-1,1}$ корневых помеченных деревьев с $q_{i}$ вершинами. Следовательно,

$$
\begin{aligned}
& \mathbf{P}\left(h\left(F_{n, N}\right)>x\right) \\
=\sum_{q_{1}+\ldots+q_{N}=n+N} \mathbf{P}\left(h\left(F_{n, N}\right)>x|| T_{i} \mid=q_{i}, 1 \leqslant\right. & i \leqslant N) \\
& \times \mathbf{P}\left(\left|T_{i}\right|=q_{i}, 1 \leqslant i \leqslant N\right) \\
= & \sum_{q_{1}+\ldots+q_{N}=n+N}\left[1-\prod_{k=1}^{N} \mathbf{P}\left(h\left(T_{k}\right) \leqslant x|| T_{k} \mid=q_{k}\right)\right] \\
& \times \mathbf{P}\left(\left|T_{i}\right|=q_{i}, 1 \leqslant i \leqslant N\right) \\
\leqslant \sum_{q_{1}+\ldots+q_{N}=n+N}\left(\sum_{k=1}^{N} \mathbf{P}\left(h\left(F_{q_{k}-1,1}\right)>x\right)\right) & \\
& \times \mathbf{P}\left(\left|T_{i}\right|=q_{i}, 1 \leqslant i \leqslant N\right),
\end{aligned}
$$

где при выводе последнего неравенства мы воспользовались тем, что при условии $\left|T_{k}\right|=q_{k}$ высота дерева $T_{k}$ имеет такое же распределение, как и распределение высоты дерева, выбранного случайно и равновероятно из множества $\mathcal{F}_{q_{k}-1,1}$. Используя (8), видим, что

$$
\begin{aligned}
\mathbf{E} h\left(F_{n, N}\right) \leqslant \sum_{q_{1}+\ldots+q_{N}=n+N}\left(\sum_{k=1}^{N} \int_{0}^{n} \mathbf{P}\left(h\left(F_{q_{k}-1,1}\right)>x\right) d x\right) \\
\times \mathbf{P}\left(\left|T_{i}\right|=q_{i}, 1 \leqslant i \leqslant N\right)
\end{aligned}
$$




$$
\begin{aligned}
& =\sum_{q_{1}+\ldots+q_{N}=n+N} \sum_{k=1}^{N} \mathbf{E}\left[h\left(F_{q_{k}-1,1}\right)\right] \mathbf{P}\left(\left|T_{i}\right|=q_{i}, 1 \leqslant i \leqslant N\right) \\
& \leqslant C \sum_{q_{1}+\ldots+q_{N}=n+N}\left(\sum_{k=1}^{N} \sqrt{q_{k}}\right) \mathbf{P}\left(\left|T_{i}\right|=q_{i}, 1 \leqslant i \leqslant N\right) .
\end{aligned}
$$

Так как $q_{1}+\ldots+q_{N}=n+N$, то

$$
\sum_{k=1}^{N} \sqrt{q_{k}} \leqslant N \sqrt{\frac{n+N}{N}}=\sqrt{N(n+N)},
$$

что в сочетании с (9) доказывает (7). Лемма доказана.

Далее будем считать, что $H_{n, N}(k)=0$, если $k \notin[0, n]$. С учетом этого соглашения введем непрерывную функцию $\hat{H}_{n, N}(x), x \in[0, \infty)$, полагая

$$
\hat{H}_{n, N}(x):=H_{n, N}(k)+\left(H_{n, N}(k+1)-H_{n, N}(k)\right)(x-k)
$$

при всех $k=0,1, \ldots$ и $x \in[k, k+1)$. Поскольку

$$
\begin{aligned}
\int_{k}^{k+1} x \hat{H}_{n, N}(x) d x= & \frac{1}{2}\left(k H_{n, N}(k)+(k+1) H_{n, N}(k+1)\right) \\
& +\frac{1}{6}\left(H_{n, N}(k)-H_{n, N}(k+1)\right)
\end{aligned}
$$

и

$$
\begin{aligned}
& \sum_{k=0}^{n}\left(k H_{n, N}(k)+(k+1) H_{n, N}(k+1)\right)=2 \sum_{k=0}^{n} k H_{n, N}(k), \\
& \sum_{k=0}^{n}\left(H_{n, N}(k)-H_{n, N}(k+1)\right)=N
\end{aligned}
$$

TO

$$
\begin{aligned}
\int_{0}^{\infty} & x \hat{H}_{n, N}(x) d x=\int_{0}^{n+1} x \hat{H}_{n, N}(x) d x \\
= & \sum_{k=0}^{n} \int_{k}^{k+1} x \hat{H}_{n, N}(x) d x=2 \sum_{k=0}^{n} k H_{n, N}(k)=2 \Upsilon\left(F_{n, N}\right)+N
\end{aligned}
$$


Для формулировки следующей леммы нам понадобится процесс $\mathcal{X}:=\left\{X_{l, t, r}, r \geqslant 0\right\}$, который имеет распределение, задаваемое соотношением

$$
\left\{X_{l, t, r}, r \geqslant 0\right\} \stackrel{d}{=}\left\{L_{t, r}^{|\mathrm{br}|}, r \geqslant 0 \mid 2 L_{t, 0}^{|\mathrm{br}|}=l\right\} .
$$

Детальное описание свойств процесса $\mathcal{X}$ можно найти в [13].

Основой наших дальнейших рассуждений будет следующее утверждение (см. следствие 10 работы [13]).

Лемма 5. Если $n$ и $N=N(n)$ меняются так, что $2 N n^{-1 / 2} \rightarrow l \in$ $[0, \infty)$ nрu $n \rightarrow \infty$, mo

$$
\left\{\frac{2}{\sqrt{n}} \hat{H}_{n, N}(2 \sqrt{n} r), r \geqslant 0\right\} \Longrightarrow\left\{X_{l, 1, r}, r \geqslant 0\right\},
$$

где символ $\Longrightarrow$ обозначает слабую сходимость в пространстве $C[0, \infty)$ непрерывных функиий, снабженном равномерной метрикой.

Заметим теперь, что высоту леса $F_{n, N}$ можно можно задать формулой

$$
h\left(F_{n, N}\right)=\min \left\{1 \leqslant k \leqslant n: H_{n, N}(k)=0\right\} .
$$

Из леммы 5 и принципа инвариантности Донскера - Прохорова следует, что при выполнении условия (4)

$$
\frac{2}{\sqrt{n}} h\left(F_{n, N}\right) \stackrel{d}{\rightarrow} \inf \left\{r>0: X_{l, 1, r}=0\right\}=: \zeta .
$$

Тот факт, что случайная величина $\zeta$ имеет собственное распределение, вытекает из непрерывности с вероятностью 1 траекторий броуновского моста $\mathcal{B}^{|\mathrm{br}|, 1}(l)$ на интервале $[0,1]$.

Лемма 5 позволяет убедиться в справедливости следующего утверждения.

Лемма 6. В условиях леммы 5

$$
\frac{1}{2 n^{3 / 2}} \Upsilon\left(F_{n, N}\right) \stackrel{d}{\rightarrow} \int_{0}^{1}\left|B_{s}^{\mathrm{br}, 1}(l)\right| d s, \quad n \rightarrow \infty .
$$

Доказательство. Поскольку

$$
\begin{aligned}
& \int_{0}^{\infty} x \hat{H}_{n, N}(x) d x=\int_{0}^{h\left(F_{n, N}\right)} x \hat{H}_{n, N}(x) d x \leqslant h\left(F_{n, N}\right) \int_{0}^{h\left(F_{n, N}\right)} \hat{H}_{n, N}(x) d x \\
& =h\left(F_{n, N}\right)\left(\sum_{k=0}^{h\left(F_{n, N}\right)}\left(H_{k, N}+\frac{1}{2}\left(H_{k+1, N}-H_{k, N}\right)\right)\right)=h\left(F_{n, N}\right)(n+N),
\end{aligned}
$$


TO

$$
\begin{aligned}
& \lim _{M \rightarrow \infty} \lim _{n \rightarrow \infty} \mathbf{P}\left(\frac{1}{n^{3 / 2}} \int_{0}^{\infty} x \hat{H}_{n, N}(x) d x>M\right) \\
&= \lim _{M \rightarrow \infty} \lim _{n \rightarrow \infty} \mathbf{P}\left(\frac{1}{n^{3 / 2}} \int_{0}^{h\left(F_{n, N}\right)} x \hat{H}_{n, N}(x) d x>M\right) \\
& \leqslant \lim _{M \rightarrow \infty} \lim _{n \rightarrow \infty} \mathbf{P}\left(\frac{1}{n^{1 / 2}} h\left(F_{n, N}\right)>M\right)=0
\end{aligned}
$$

Зафиксируем $M>0$ и, используя соотношение (10), запишем представление

$$
\begin{gathered}
\frac{1}{2 n^{3 / 2}}\left(\Upsilon\left(F_{n, N}\right)+N\right)=\frac{1}{2 n^{3 / 2}} \int_{0}^{\infty} x \hat{H}_{n, N}(x) d x=\int_{0}^{\infty} r\left(\frac{2}{\sqrt{n}} \hat{H}_{n, N}(2 \sqrt{n} r)\right) d r \\
\quad=\int_{0}^{M} r\left(\frac{2}{\sqrt{n}} \hat{H}_{n, N}(2 \sqrt{n} r)\right) d r+\frac{1}{2 n^{3 / 2}} \int_{2 M \sqrt{n}}^{\infty} x \hat{H}_{n, N}(x) d x .
\end{gathered}
$$

В силу (12) и (15)

$$
\begin{aligned}
\lim _{M \rightarrow \infty} \lim _{n \rightarrow \infty} \mathbf{P}\left(\int_{2 M \sqrt{n}}^{\infty} x \hat{H}_{n, N}(x) d x>0\right) \\
=\lim _{M \rightarrow \infty} \lim _{n \rightarrow \infty} \mathbf{P}\left(h\left(F_{n, N}\right)>2 M \sqrt{n}\right)=0 .
\end{aligned}
$$

Поэтому при больших значениях $M$ интерес представляет лишь первое слагаемое в правой части (16).

Из леммы 5 и принципа инвариантности Донскера - Прохорова вытекает, что для каждого $M>0$

$$
\int_{0}^{M} r\left(\frac{2}{\sqrt{n}} \hat{H}_{n, N}(2 \sqrt{n} r)\right) d r \stackrel{d}{\rightarrow} \int_{0}^{M} r X_{l, 1, r} d r .
$$

Устремляя $M$ к бесконечности и вспоминая (4) и (17), приходим к соотношению

$$
\frac{1}{2 n^{3 / 2}} \Upsilon\left(F_{n, N}\right) \stackrel{d}{\rightarrow} \int_{0}^{\infty} r X_{l, 1, r} d r .
$$

Для завершения доказательства осталось заметить, что в силу (11)

$$
\int_{0}^{\infty} r X_{l, 1, r} d r \stackrel{d}{=} \int_{0}^{1}\left|B_{s}^{\mathrm{br}, 1}(l)\right| d s .
$$

Лемма доказана. 


\section{3. Доказательство теоремы}

Обозначим $\rho_{i}$ корень дерева $T_{i}$ и введем набор

$$
\left\{Q_{u}, u \in F_{n, N}, v \neq \rho_{i}, i=1,2, \ldots, N\right\},
$$

состоящий из независимых случайных величин, имеющих равномерное распределение на отрезке $(-1 / 2,1 / 2)$. С каждой вершиной $u \neq \rho_{i}$ свяжем случайную величину

$$
W(u):=\left\lfloor\left(Q_{u}+\frac{1}{2}\right) m_{u}\right\rfloor,
$$

где $\lfloor x\rfloor$ - целая часть числа $x$. Ясно, что случайная величина $W(u)$ имеет равновероятное распределение на множестве $0,1, \ldots, m_{u}-1$. Положим

$$
\mathcal{I}^{*}\left(T_{i}\right):=\sum_{u \in T_{i}, u \neq \rho_{i}}\left(W(u)-\mathbf{E}\left[W(u) \mid T_{i}\right]\right)
$$

и рассмотрим случайную величину

$$
\mathcal{I}^{*}\left(F_{n, N}\right):=\sum_{i=1}^{N} \mathcal{I}^{*}\left(T_{i}\right)=\sum_{i=1}^{N} \sum_{u \in T_{i}, u \neq \rho_{i}}\left(W(u)-\mathbf{E}\left[W(u) \mid T_{i}\right]\right) .
$$

В силу (3) и леммы 2 величина $\mathcal{I}^{*}\left(F_{n, N}\right)$ совпадает по распределению с центрированным числом инверсий в лесе $F_{n, N}$ без учета инверсий, порождаемых корнями деревьев $T_{1}, \ldots, T_{N}$. Поскольку

$$
\mathcal{I}\left(F_{n, N}\right)-\frac{1}{2} \Upsilon\left(F_{n, N}\right)=\sum_{i=1}^{N} \sum_{u \in T_{i}}\left(W(u)-\mathbf{E}\left[W(u) \mid T_{i}\right]\right)
$$

и

$$
\sum_{i=1}^{N}\left(W\left(\rho_{i}\right)-\mathbf{E}\left[W\left(\rho_{i}\right) \mid T_{i}\right] \leqslant n\right.
$$

TO

$$
\mathcal{I}^{*}\left(F_{n, N}\right) \leqslant \mathcal{I}\left(F_{n, N}\right)-\frac{1}{2} \Upsilon\left(F_{n, N}\right) \leqslant \mathcal{I}^{*}\left(F_{n, N}\right)+n .
$$

Заметим далее, что для любой вершины $u \in T_{i}$

$$
\begin{aligned}
W(u)-\mathbf{E}\left[W(u) \mid T_{i}\right] & \leqslant\left(Q_{u}+\frac{1}{2}\right) m_{u}-\mathbf{E}\left[\left(Q_{u}+\frac{1}{2}\right) m_{u}-1\right] \\
& =Q_{u} m_{u}+1
\end{aligned}
$$


И

$$
\begin{aligned}
W(u)-\mathbf{E}\left[W(u) \mid T_{i}\right] & \geqslant\left(Q_{u}+\frac{1}{2}\right) m_{u}-1-\mathbf{E}\left[\left\lfloor\left(Q_{u}+\frac{1}{2}\right) m_{u}\right\rfloor\right] \\
& \geqslant Q_{u} m_{u}-1
\end{aligned}
$$

Положим

$$
\mathcal{J}\left(F_{n, N}\right)=\sum_{i=1}^{N} \sum_{u \in T_{i}, u \neq \rho_{i}} Q_{u} m_{u}
$$

Из приведенных выше оценок вытекает, что

$$
\mathcal{I}^{*}\left(F_{n, N}\right) \leqslant \sum_{i=1}^{N} \sum_{u \in T_{i}, u \neq \rho_{i}}\left(Q_{u} m_{u}+1\right) \leqslant \mathcal{J}\left(F_{n, N}\right)+n
$$

и

$$
\mathcal{I}^{*}\left(F_{n, N}\right) \geqslant \mathcal{J}\left(F_{n, N}\right)-n
$$

Следовательно,

$$
\mathcal{J}\left(F_{n, N}\right)-2 n \leqslant \mathcal{I}\left(F_{n, N}\right)-\frac{1}{2} \Upsilon\left(F_{n, N}\right) \leqslant \mathcal{J}\left(F_{n, N}\right)+2 n .
$$

Ясно, что $\mathbf{E} J\left(F_{n, N}\right)=0$ и

$$
\begin{aligned}
\mathbf{E} \mathcal{J}^{2}\left(F_{n, N}\right) & =\mathbf{E}\left(\sum_{i=1}^{N} \sum_{u \in T_{i}, u \neq \rho_{i}} Q_{u} m_{u}\right)^{2} \\
& =\sum_{i, j=1}^{N} \mathbf{E}\left(\sum_{u(i) \in T_{i}, u(i) \neq \rho_{i}} \sum_{u(j) \in T_{j}, u(j) \neq \rho_{j}} Q_{u(i)} Q_{u(j)} m_{u(i)} m_{u(j)}\right) \\
& \leqslant \sum_{i, j=1}^{N} \mathbf{E}\left(\sum_{u(i) \in T_{i}} \sum_{u(j) \in T_{j}} \mathbf{E}\left[Q_{u(i)} Q_{u(j)} \mid T_{i}, T_{j}\right] m_{u(i)} m_{u(j)}\right) .
\end{aligned}
$$

Поскольку случайные величины $Q_{u(i)}$ и $Q_{u(j)}$ зависимы тогда и только тогда, когда $u(i)=u(j)$, и кроме того,

$$
\mathbf{E}\left[Q_{u} \mid T\right]=0, \quad \mathbf{E}\left[Q_{u}^{2} \mid T\right]=\int_{-1}^{1} x^{2} d x=\frac{2}{3}
$$

для любого дерева $T$ и любой вершины $u \in T$, то

$$
\mathbf{E} \mathcal{J}^{2}\left(F_{n, N}\right) \leqslant \frac{2}{3} \sum_{i=1}^{N} \mathbf{E}\left(\sum_{u \in T_{i}} m_{u}^{2}\right) .
$$


Для не обязательно различных вершин $v_{1}, \ldots, v_{k}$ дерева $T$ положим

$$
c\left(v_{1}, \ldots, v_{k}\right):=\left|\left\{u \in T: u \preceq v_{1}, \ldots, u \preceq v_{k}\right\}\right| .
$$

Несложно проверить, что

$$
\begin{aligned}
\sum_{u \in T} m_{u}^{2} & =\sum_{u \in T}\left(\sum_{u \preceq v} I\{u \preceq v\}\right)^{2} \\
& =\sum_{u \in T} \sum_{u \preceq v_{1}, u \preceq v_{2}} I\left\{u \preceq v_{1}, u \preceq v_{2}\right\}=\sum_{v_{1}, v_{2} \in T} c\left(v_{1}, v_{2}\right) \\
& \leqslant|T|^{2}(h(T)+1) .
\end{aligned}
$$

Отсюда и из леммы 4 следует, что найдется такая константа $C$, что

$$
\begin{aligned}
\mathbf{E} \mathcal{J}^{2}\left(F_{n, N}\right) & \leqslant \frac{2}{3} \mathbf{E}\left[\sum_{i=1}^{N}\left|T_{i}\right|^{2}\left(h\left(T_{i}\right)+1\right)\right] \leqslant \frac{2}{3} \mathbf{E}\left[\left|F_{n, N}\right|^{2}\left(h\left(F_{n, N}\right)+1\right)\right] \\
& \leqslant \frac{2}{3} n^{2} \mathbf{E}\left[h\left(F_{n, N}\right)+1\right] \leqslant C n^{2} \sqrt{N n}
\end{aligned}
$$

при всех $n=1,2, \ldots$ и $N \leqslant n$. Учитывая условие (4), заключаем, что найдется константа $C_{3}$, для которой

$$
\mathbf{E} \mathcal{J}^{2}\left(F_{n, N}\right) \leqslant C_{3} n^{11 / 4}
$$

при всех $n \geqslant 1$. Отсюда и из (18) выводим, что

$$
\begin{aligned}
\mathbf{P}\left(\mid \frac{1}{n^{3 / 2}}\right. & \left.\mathcal{I}\left(F_{n, N}\right)-\frac{1}{2 n^{3 / 2}} \Upsilon\left(F_{n, N}\right) \mid \geqslant \frac{2}{n^{1 / 16}}\right) \\
& =\mathbf{P}\left(\left|\mathcal{I}\left(F_{n, N}\right)-\frac{1}{2} \Upsilon\left(F_{n, N}\right)\right| \geqslant 2 n^{23 / 16}\right) \\
& \leqslant \mathbf{P}\left(\left|\mathcal{J}\left(F_{n, N}\right)\right|+2 n \geqslant 2 n^{23 / 16}\right) \leqslant \mathbf{P}\left(\left|\mathcal{J}\left(F_{n, N}\right)\right| \geqslant n^{23 / 16}\right) \\
& \leqslant \frac{\mathbf{E} \mathcal{J}^{2}\left(F_{n, N}\right)}{n^{23 / 8}} \leqslant \frac{C_{3} n^{11 / 4}}{n^{23 / 8}}=\frac{C_{3}}{n^{1 / 8}} \rightarrow 0
\end{aligned}
$$

при $n \rightarrow \infty$. Последнее в сочетании с (14) показывает, что если параметры $N, n$ стремятся к бесконечности так, что $2 N n^{-1 / 2} \rightarrow l \in[0, \infty)$, то

$$
\frac{2}{n^{3 / 2}} \mathcal{I}\left(F_{n, N}\right) \stackrel{d}{\rightarrow} \int_{0}^{1}\left|B_{s}^{\mathrm{br}, 1}(l)\right| d s .
$$

Теорема доказана.

Автор благодарен рецензенту, стимулирующие вопросы которого позволили улучшить изложение представленных здесь результатов. 


\section{Список литературы}

[1] Ватутин В.А., "Распределение расстояния до корня минимального поддерева, содержащего все вершины данной высоты”, Теория вероятн. и ее примен., 38:2 (1993), 273-287.

[2] Ватутин В.А., “Асимптотические свойства числа инверсий в раскрашенных деревьях", Математические вопросы криптографии, 10:4 (2019), 9-24.

[3] Харрис Т., Теория ветвящихся процессов, М.: Наука, 1966, 355 с.

[4] Колчин В.Ф., Случайные отображения, М.: Наука, 1984, 207 с.

[5] Павлов Ю.Л., "Предельные распределения высоты случайного леса", Теория вероятн. и ее примен., 28:3 (1983), 449-457.

[6] Addario-Berry L., Devroye L., Janson S., "Sub-Gaussian tail bounds for the width and height of conditioned Galton-Watson trees", Ann. Probab., 41:2 (2013), 1072-1087.

[7] Aldous D., Pitman J., "Brownian bridge asymptotics for random mappings", Random Struct. Algor., 5 (1994), 487-512.

[8] Cai X. S., Holmgren C., Janson S., Johansson T., Skerman F., "Inversions in split trees and conditional Galton-Watson trees", Comb., Probab. and Comput., 28:3 (2019), 335-364.

[9] Flajolet P., Poblete P., Viola A., "On the analysis of linear probing hashing", Algorithmica, 22 (1998), 490-515.

[10] Chassaing P., Louchard G., "Reflected Brownian bridge area conditional on its local time at the origin", J. Algorithms, 44 (2002, 29-51).

[11] Knight F. B., "The moments of the area under reflected Brownian bridge conditional on its local time at zero", Int. J. Stoch. Analysis, 13:2 (2000), 99-124.

[12] Pitman J., "Combinatorial Stochastic Processes", Lect. Notes Math., 1875, SpringerVerlag Berlin Heidelberg, 2006, 260 pp.

[13] Pitman J., "The SDE solved by local times of a Brownian excursion or bridge derived from the height profile of a random tree or forest", Ann. Probab., 27:1 (1999), 261-283. 